\title{
LA FUERZA EN EL CURRICULUM DE LA EDUCACIÓN FÍSICA ES COLAR Un análisis del papel de la fuerza en el vitae actual desde la perspectiva del desarrollo
}

\section{AUTORES}

ONOFRE RICARDO CONTRERAS JORDÁN CATEDRÁTICO DE DIDÁCTICA DE LA EDUCACTÓN FÍSICA FACULTAD DE CIENCIAS DEL DEPORTE DE TOLEDO UCLM LUIS MIGUEL GARCÍA LÓPEZ PROFESOR DE DIDÁCTICA DE LA EDUCACIÓN FÍSICA ESCUELA DE MAGISTERTO DE TOLEDO UCLM
DAVID GUTIÉRREZ DÍAZ DEL CAMPO PROFESOR DE DIDÁCTICA DELA EOUCACTÓN FÍSICA ESCUELA DE MAGISTERTO DE CUENCA, UCLM

\section{REFERENCIAS FUNDAMENTALES DEL TRABAJO DE FUERZA}

El entrenamiento de la fuerza en las edades tempranas ha estado sometido a una serie de creencias que hacían concebir dicha actividad como naturalmente contraproducente desde el punto de vista de una correcta formación. Dichas creencias fueron más tarde alimentadas por investigaciones como las de la Academia Ame ricana de Pediatría o el estudio de Vrijens citado por García Manso (1999:463)en las que se ponía de manifiesto que la mayoría de los grupos testados no ofrecía modificaciones significativas en relación al aumento de la fuerza.

Sin embargo, García Manso (1999:464) a la vez que advierte de los errores metodológicos de la investigación de Vrijens, nos da cuenta de hallazgos más actua les en las que se demuestra claramente que no sólo aumenta la fuerza sometida a un entrenamiento riguroso, sino que además se incrementa de manera especial en los estadios prepuberales más incluso que en puberales y postpuberales.

En efecto, ante la tesis de que el entrenamiento de fuerza antes de los 10 años ( 8 en las niñas) no produce ningún efecto se ha constatado que algunos deportistas que la requieren la desarrollan (gimnasia artística) aunque no por vía hipertrófica. En este sentido en la fase anterior a la adolescencia las ganancias de fuerza en valores relativos son más importantes que en valores absolutos.

Comienza así un camino de retorno que se dirige eliminar la mala prensa del trabajo de fuerza en la escuela y contrariamente sentar las bases que promuevan una formación de este tipo riguroso en el ámbito escolar. No obstante, hay que hacer notar que genéticamente el organismo desarrolla la capacidad de fuerza muscular máxima después de haber solidificado el crecimiento y completado el proceso de desarrollo sexual de ahi que sea durante la adolescencia cuando se verifican las condiciones morfofuncionales y psicológicas favorables para el desarrollo de la fuerza máxima.
De cara a una buena programación para el desarrollo de la fuerza se han de tener en cuenta dos referencias básicas, por una parte la edad, o mejor dicho la maduración de la que la edad sólo es una referencia estandarizada, y por otra, el crecimiento del sujeto. Ambas referencias se deben poner en relación con lo que se ha venido denominando "fases sensibles" para el fuerza.

En efecto, cuando hablamos de "fase sensible", nos cada una de las capacidades físicas son diferentes, de modo que se acepta por una parte de la comunidad científica que existen unos períodos durante los cuales el organismo es especialmente receptivo para el desarrollo de una capacidad, de ahí que debe aprovecharse esta etapa para optimizar el desarrollo de la capacidad físi-

Asi se ha definido la fase sensible como "aquel pe ríodo delimitado del desarrollo durante el cual los seres humanos reaccionan de modo más intenso que en otro momento ante determinados estímulos externos, dando lugar a los correspondientes efectos". Sin embargo, la comunidad científica no se muestra unánime en la existencia de las "fases sensibles" ya que mientras unos opinan que están científicamente demostradas (Hahn, 1988), otros mantienen la posición contraria (Winter, 1975, cit. por García Manso, 1999).

Algunos autores de nuestro entorno han adoptado una postura ecléctica (ver Navarro Valdivielso, 2001:140), de manera que afirman que existen ciertas etapas recomendables para llevar a cabo el entrenamiento de algunas capacidades con el fin de evitar riesgos y favorecer el afianzamiento de una reserva de entrenamiento duradera. que se ha venido denominando "fases sensibles" para el estamos refiriendo a que los ritmos de desarrollo de ca en cuestión. 
Durante la infancia el incremento de la fuerza es progresivo y suave acelerándose durante la pubertad y estabilizándose después de ella. El $50 \%$ de la fuerza se alcanza a los 10 años en las niñas y a los 12 en los niños.

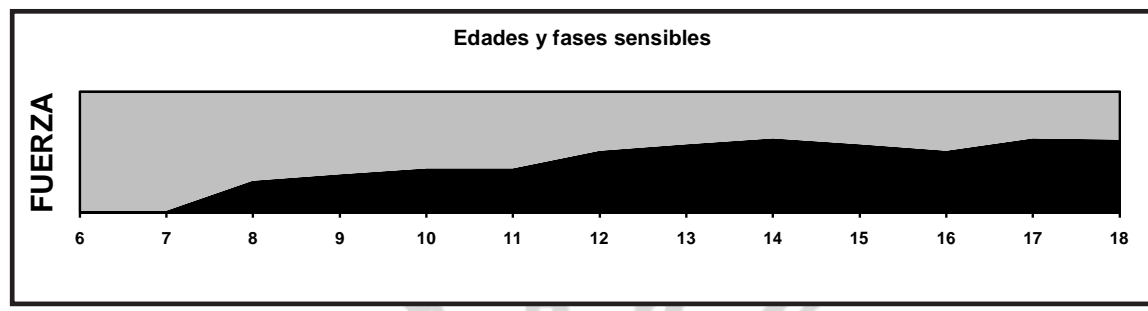

Adaptado de Fernando Valdivieso, 2001

En relación a la edad la fuerza aumenta de manera progresiva tanto en los niños como en las niñas, de manera que hasta la edad prepuberal tienen la misma fuerza ambos sexos hasta los 12 ó 13 años en que se produce la diferenciación. A partir de la pubertad los varones presentan un incremento importante de la misma por cada año de vida.

En todo caso el incremento de la fuerza se debe a dos factores, por una parte a la maduración y por otra al crecimiento del sujeto, de manera más específica a la maduración de sus sistema nerviosos y endocrino, siendo a partir de la pubertad en que se produce una explosión hormonal que no debe asociarse a la edad cronológica sino a la maduración.

Por su parte el crecimiento actúa proporcionando en cada momento de la vida unas condiciones diferentes para la mejora de las distintas manifestaciones de la fuerza de acuerdo a las siguientes etapas establecidas por García Manso (1999:478):

- De los 7 a los 10 años se produce un desarrollo lento de la fuerza.

- Durante la etapa prepuberal, la fuerza rápida y máxima muestran aumentos la te altos. muestra importantes diferencias individuales que oscilan entre un $60 \%$ y un $90 \%$.

- En la pubertad aparecen aumentos elevados de fuerza rápida y máxima que adquiere en las niñas un aspecto específico al producirse en un espacio breve y más intenso.

- El desarrollo de la fuerza durante la adolescencia tiene una evidente mejora con cuotas de aumento permanentes, especialmente, en lo que se refiere a la fuerza máxima y rápida. Sin embargo, la fuerza resistencia se desarrolla en menor medida.

Otra clasificación es la mostrada por Tschitchikin (cit. por García Manso, 1999:478) que distingue cuatro fases en el desarrollo de la fuerza:

$\square$ De los 8 a los 10 años.

口 De los 11 a los 13 "

a De los 14 a los 15

- De los 16 a los 17 "

En estos períodos la fuerza absoluta alcanza sus valores máximos después de la pubertad, es decir, entre los 15 y los 17 años, mientras que la fuerza relativa los alcanza entre los 13 y 14 años que es el período en el que los jóvenes crean las condiciones más favorables para los saltos.

Por su parte, Navarro Valdivielso (2001:152), distingue el desarrollo de las tres manifestaciones más populares de la fuerza: la fuerza máxima, la fuerza rápida o fuerza-velocidad y la fuerza-resistencia, a cuyo fin establece los siguientes períodos:

- Fuerza máxima: comienza su desarrollo a los 1416 años, mejorando durante los $16-18$ y alcanzando su máxima expresión hacia los 18-20.

- La fuerza resistencia presenta el mismo cuadro de evolución del desarrollo que la fuerza máxima.

1 Fuerza velocidad: comienza su desarrollo hacia los 12-14 años, obteniendo su máximo cota a los 14- 16 en que se mantiene constante.

Por último, podríamos establecer las siguientes recomendaciones tras las consideraciones que hemos efectuado:

$\checkmark$ El desarrollo de la fuerza máxima se puede realizar desde la infancia sin embargo no se deben utilizar los mismos medios que en la edad adulta.

$\checkmark$ El beneficio del entrenamiento no se traduce en hipertrofia muscular sino en mejora de la coordinación inter o intramuscular.

$\checkmark$ A pesar de las mayores posibilidades de mejora que podemos encontrar en el hombre respecto a la mujer, estas diferencias se reducen cuando hacemos referencia a valores relativos (incrementos por peso corporal).

1. El entrenamiento de la fuerza en niños y jóvenes.

Los objetivos del entrenamiento de fuerza en la edad escolar tienden preferentemente a situarse en una perspectiva de salud del escolar antes que como búsqueda del rendimiento de manera que podríamos situarlos en los siguientes aspectos:

$\square$ Desarrollo muscular armónico.

- Buena postura corporal.

- Adecuada adaptación muscular preventiva de lesiones.

$\checkmark$ Acceder al alto rendimiento en el futuro. 
En este sentido García Manso establece las siguientes fases:

\begin{tabular}{|c|c|c|c|}
\hline \multirow[b]{2}{*}{ FASE } & \multicolumn{2}{|c|}{ EDAD } & \multirow[b]{2}{*}{ CICLO ESCOLAR } \\
\hline & VARONES & MUJERES & \\
\hline $\begin{array}{l}\text { Inicio de la fuerza rápida y } \\
\text { Mejora del tono muscular básico }\end{array}$ & $7-8$ & $7-8$ & $\begin{array}{l}2^{\circ} \text { curso del Primer Ciclo de } \\
\text { Educación Primaria }\end{array}$ \\
\hline $\begin{array}{l}\text { Inicio al acondicionamiento muscular } \\
\text { de base al desarrollo de la fuerza }\end{array}$ & $10-11$ & $10-11$ & $\begin{array}{c}5^{\circ} \text { curso del Tercer Ciclo de } \\
\text { Educación Primaria }\end{array}$ \\
\hline $\begin{array}{c}\text { Inicio al entrenamiento de fuerza } \\
\text { máxima y fuerza resistencia de baja } \\
\text { intensidad. }\end{array}$ & $12-14$ & $12-14$ & $\begin{array}{l}1^{\circ} \text { Ciclo de la Educación } \\
\text { Secundaria Obligatoria }\end{array}$ \\
\hline $\begin{array}{l}\text { Inicio al entrenamiento de fuerza } \\
\text { resistencia de alta intensidad y } \\
\text { característica anaeróbica. }\end{array}$ & $13-15$ & $13-15$ & $\begin{array}{c}3^{\circ} \text { Curso del Segundo Ciclo de } \\
\text { la Educación Secundaria } \\
\text { Obligatoria }\end{array}$ \\
\hline $\begin{array}{l}\text { Inicio al entrenamiento de Fuerza } \\
\text { máxima neuromuscular. }\end{array}$ & $14-15$ & $14-15$ & $\begin{array}{l}\text { Segundo Ciclo de la Educación } \\
\text { Secundaria Obligatoria }\end{array}$ \\
\hline $\begin{array}{l}\text { Entrenamiento de máximo } \\
\text { rendimiento }\end{array}$ & 17 & 16 & Bachiller \\
\hline
\end{tabular}

Por su parte Navarro Valdivielso establece las tres siguientes fases:

\section{$\sqrt{ }$ Primer período (2 a 7 años).}

Se trata de adaptar el sistema músculo tendinoso a crecimiento longitudinal óseo y al nuevo esquema corporal. Se requiere una actividad física multilateral a través de juegos y ejercicios que involucren la mayor cantidad de grupos musculares que desarrollen el tono muscular.

$\sqrt{ }$ Segundo período (8 a 11 años).

Se inicia la fase sensible para comenzar a estimular específicamente la fuerza velocidad. Se deben emplear ejercicios que utilicen el peso corporal o movimientos ligeros con balones medicinales. Son ejercicios contraindicados el desarrollo de la hipertrofia y la coordinación intramuscular.

\section{$\checkmark$ Tercer período (pubertad y adolescencia).}

$\mathrm{Se}$ dan las mejores condiciones biológicas en relación a la presencia hormonal que permite el efecto anabólico proteico que origina la hipertrofia muscular. $\mathrm{Sin}$ embargo, en la fase puberal las condiciones biomecánicas son desfavorables por el excesivo y desequilibrado crecimiento óseo longitudinal.

Con respecto a la fuerza explosiva, cuya etapa sensible se situaba en el período anterior, en este se puede continuar su entrenamiento. Los ejercicios en forma de juego que contemplan el empleo de pequeñas sobrecargas, juegos de transporte y de lucha, así como circuitos parecen ser los medios más indicados para niños de 10 a 14 años.

La fuerza resistencia, cuya fase sensible se sitúa en este período, se debe comenzar a trabajar con ejercicios que impliquen grandes músculos para luego ejercitar la musculatura más específica. El trabajo recomendado es de tres veces por semana con una intensidad de 10 a 25 repeticiones realizando tres series.
dado es de tres veces por semana con una inten

Finalmente, el desarrollo de la fuerza máxima por vía de la coordinación intramuscular deberá comenzar en una fase más avanzada, debido a las altas cargas que se han de emplear.
1. El trabajo de fuerza en el currículum de Educación Física. Análisis crítico.

Se trata de ver en este momento cómo incorporan los distintos diseños curriculares del Estado español en las diferentes Etapas educativas la teorización científica antes comentada, a fin de que podamos establecer una perspectiva comparativa que nos permita un análisis crítico del tratamiento de la fuerza en el currículum escolar.

1.1. La ausencia de referencia directa a la fuerza en el currículum de Educación Primaria.

- Las únicas referencias no expresas al trabajo de fuerza en la Educación Primaria están incluidas en las referencias a la salud que se expresa así en los objetivos generales del área:

- Adoptar hábitos de higiene, de alimentación, de posturas y de ejercicio físico, manifestando una actitud responsable hacia su propio cuerpo y de respeto a los demás, relacionando estos hábitos con los efectos sobre la salud.

De forma que se establece un bloque de contenidos específico denominado Salud Corporal que cobra una mayor importancia a medida que se avanza en la Etapa alcanzando su pleno sentido en el tercer ciclo. Hay que indicar, por otra parte, que tiene una gran relación con el resto de bloques a los que añade una importante reflexión sobre los efectos de la actividad física en su propio cuerpo y en su salud para que la actividad física tenga un sentido de utilidad. En todo caso, el sentido final debe ser el de aprender actitudes y valores.

Tal vez la referencia más directa sea la contenida en los criterios de evaluación en los que se establece:

- Haber incrementado globalmente las capacidades físicas básicas de acuerdo con el momento de desarrollo motor, acercándose a los valores normales del grupo de edad en el entorno de referencia.

A la vista de lo expuesto podemos afirmar que el currículum de Educación Primaria no se plantea el tra- 
bajo de fuerza en esta Etapa cuando a la vista de las consideraciones previas efectuadas seria el momento más adecuado para trabajarla del modo siguiente:

- En el primer ciclo (6 a 8 años) realizar un trabajo de "iniciación a la fuerza rápida y mejora del tono muscular básico".

- En el tercer ciclo (10 a 12 años) se deberían hacer actividades de cara a la "iniciación al acondicionamiento muscular de base al desarrollo de la fuerza".

- Es preciso tener en cuenta que durante estos dos ciclos se inicia la fase sensible de la fuerza velocidad por lo que resultan de aplicación las recomendaciones sobre el trabajo anteriormente efectuadas.

1.2. Las primeras referencias a la mejora de la condición física. La ESO.

La propia introducción del Real Decreto de enseñanza mínimas justifica la Educación Física porque al hombre del siglo XXI le resulta imprescindible "la ejercitación del cuerpo si no quiere ver atrofiadas prematuramente muchas de sus funciones y capacidades corporales". En este sentido los objetivos 4 y 5 establecen:

- Realizar tareas dirigidas a la mejora de la condición física y las condiciones de salud y calidad de vida, haciendo un tratamiento discriminado de cada capacidad.

- Incrementar sus posibilidades de rendimiento motor mediante el acondicionamiento y mejora de las capacidades físicas.

A tal fin establecen tan sólo dos contenidos que son:

- Condición física y salud.

- Habilidades específicas

El primero de ellos que es el que nos interesa en relación a la fuerza hemos de comentar que la condición física se liga a la salud, por lo que debemos entender que se aleja del rendimiento para desarrollar las capacidades físicas desde un punto de vista higiénico. Por otra parte, ambos contenidos vienen secuenciados por cursos, lo que supone la existencia de un currículum más cerrado y la consiguiente perdida de autonomía del profesor en el diseño de la materia.

En el marco descrito se plantean los siguientes aprendizajes en relación a la fuerza:

\section{Primer Curso.}

Condición física y salud.

- Condición física. Concepto. Capacidades físicas relacionadas con la salud.

- Levantamiento y transporte de cargas pesadas (carteras).

- Acondicionamiento general de las capacidades físicas relacionadas con la salud. Práctica de carrera continua, juegos, ejercicios dinámicos de fuerza general y estiramientos.

$\square$ Fortalecimiento de la musculatura de sostén mediante la realización de ejercicios de movilidad articular, de fortalecimiento muscular y de relajación.
Por lo que se refiere a los criterios de evaluación tan sólo se menciona uno de carácter genérico que dice así:

- "Incrementar las capacidades físicas relacionadas con la salud, trabajadas durante el curso: mejorando con respecto a su nivel de práctica".

El tratamiento de la fuerza que se realiza en este primer curso nos sugiere el siguiente comentario. La edad de los alumnos es la de trece años o catorce años, es decir, es el momento en que se dan las mejores condiciones biológicas para su desarrollo dada la presencia hormonal que permite la hipertrofia muscular. Asimismo, como hemos visto con anterioridad la edad que tratamos admite el trabajo específico de algunas manifestaciones de la fuerza, como es la fuerza velocidad mientras que el diseño curricular sólo se refiere al trabajo de fuerza general. Por otra parte, el levantamiento y transporte de cargas pesadas está más en relación a la ría ser considerado con más propiedad en la Educación Primaria que en la Secundaria Obligatoria. Finalmente, por lo que se refiere a la evaluación hay que indicar que no parece un criterio muy adecuado pues la mejora de la fuerza con el trabajo prescrito no se producirá y en caso de hacerlo será debido más al desarrollo natural de los alumnos que como consecuencia del trabajo realizado.

Segundo Curso

Condición física y salud.

- Toma de conciencia de la propia condición física y predisposición a mejorarla con un trabajo adecuado.

Por lo que se refiere a los criterios de evaluación tampoco existen tanto más en este caso que está claramente referido al aprendizaje de una actitud.

No existe ninguna referencia concreta a la fuerza en sus diferentes manifestaciones, lo que supone que tal tipo de trabajo no ha sido previsto por el legislador, sobre todo teniendo en cuenta la detallada secuenciación por cursos que se ha impuesto.

\section{Tercer Curso.}

Condición física y salud.

No existe contenido de enseñanza-aprendizaje específico ni criterios de evaluación.

No existe ninguna referencia concreta a la fuerza en sus diferentes manifestaciones, lo que supone que tal tipo de trabajo no ha sido previsto por el legislador, sobre todo teniendo en cuenta la detallada secuenciación por cursos que se ha impuesto.

Cuarto Curso.

Condición física y salud.

- Capacidades físicas relacionadas con la salud: fuerza y resistencia muscular. Concepto. Manifestaciones básicas. Tipos de ejercicios.

- Acondicionamiento de la fuerza resistencia mediante prácticas de ejercicios localizados y en parejas. Elaboración de un repertorio de ejercicios de fuerza.

Efectos del trabajo de fuerza sobre el estado de salud. 
Por lo que se refiere a los criterios de evaluación se han establecido los siguientes:

- Incrementar las capacidades físicas de resisten cia, flexibilidad y fuerza y resistencia muscular respecto a sí mismo y al entorno de referencia.

- Analizar los efectos duraderos que son beneficiosos para la salud del trabajo de fuerza y resistencia muscular.

Los 16 o 17 años constituyen un momento bastante tardío para el inicio del trabajo de la fuerza resistencia que se debía de haber iniciado hacia el primer ciclo de la ESO tanto en baja con en alta intensidad, asimismo resulta también un poco tarde para el inicio de la fuerza máxima, ya que en esta edad es posible comenzar el entrenamiento de máximo rendimiento, y no es que olvide mos la premisa inicial del trabajo de la condición física ligado a la salud, sino que dicho trabajo podía haber sido mejorado a través de un tratamiento específico de las distintas manifestaciones de la fuerza. A estos efectos, no resultara extraño que el alumnado mejore las capacidades establecidas por los criterios de evaluación ya que el moment otra cosa.

4.3. Autonomía en la planificación y evaluación del trabajo de fuerza. El Bachillerato.

Durante el Bachillerato, la Introducción del Rea Decreto de enseñanza mínimas establece claramente do ejes de actuación. Uno de ellos, el que a nosotros nos interesa en este momento, es la mejora de la salud, entendida no sólo como ausencia de enfermedad, sino como responsabilidad individual y como construcción social. En esta concepción tienen cabida el desarrollo de componentes saludables de la condición física y la adopcion de actitudes críticas ante prácticas que inciden negativamente en las mismas.

En esta etapa, y teniendo en cuenta las finalidades atribuidas al Bachillerato, este proceso debe contribuir también a consolidar la autonomía plena del alumnado para satisfacer sus propias necesidades motrices. Para conseguir este empeño es necesario completar los conocimientos referidos al saber con los relativos al "saber hacer", de forma que teoría y práctica constituyan a esencia de la Educación Física, propiciando en los alum nos la adquisición de los procedimientos que le son necesarios para planificar, organizar y dirigir sus propias actividades, sin olvidar nunca su marcado carácter procedimental. En este sentido se establece el objetivo general de área número 2:

- Evaluar el nivel de condición física y elaborar y poner en práctica un programa de actividad fís cay salud incrementando las capacidades implicadas.

A tal fin establece el contenido siguiente

$\square$ Condición física y salud.

- Realización de pruebas de evaluación de la condición física saludable.

- Práctica de sistema y ejercicios para el desarrollo de las capacidades físicas relacionadas con la salud. Principios y factores a considerar.

- Aceptación de la responsabilidad en el mantenimiento y mejora de la condición física.

- Elaboración y puesta en práctica, de manera autónoma, de un programa personal de actividad física y salud, atendiendo a la frecuencia, intensidad, tiempo y tipo de actividad.

Por lo que se refiere a los criterios de evaluación, los que revisten alguna relevancia en el tema de la fuerza son:
- Mejorar las capacidades físicas relacionadas con la salud acercándose a los valores normales del entorno de referencia.

- Realizar de manera autónoma un programa de actividad física y salud, utilizando las variables de frecuencia, intensidad, tiempo y tipo de actividad.

A la vista de todo lo expuesto el alumnado en esta Etapa debe conocer las distintas manifestaciones de la fuerza, así como los medios y ejercicios adecuados par ración ración, e intensidad a fin de que sean capaces de coneccionar su propio programa de trabajo en el que han de ser incluidos aquellos instrumentos que le permitan una evaluación de dicho trabajo. Sin embargo, aunque la sugerencia es muy interesante el tiempo semanal dedicado a la Educación Fisica imposibilita tan loables proyectos que sin duda habrán de quedar, en el mejor de os casos, como meros aprendizajes teoricos a los que habra de faltar el hábito y la actitud positiva hacia el ejercicio físico.

\section{Conclusiones finales.}

En general, el diseño curricular de la fuerza en la escuela resulta bastante pobre de manera que podemos evaluar como escaso el acierto del legislador en este sentido, ya que en el mismo no se han tenido en cuenta ilas fases sensibles" nilas diferentes etapas que planrollo de las distintas manifestaciones de la fuerza.

Todo ello ha conducido a realizar una inadecuada secuenciación que olvida los anteriores aspectos, de

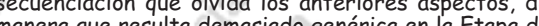
manera que resulta demasiado genérica en la Etapa de Educacion Primaria ya que no desciende al trabajo concreto y demasiado puntual en la Etapa de Educación crecun aspectos especificos de cada es de la fuerza

Asimismo, debemos consignar el escaso tiempo que se dedica a la Educación Física semanalmente lo que impide un adecuado trabajo que seguramente sólo puede afrontar con cierto rigor la creación de hábitos de actividad física ya que con la dedicación establecida es muy difícil establecer algún tipo de rendimiento cuestión extraordinariamente interesante en torno a desvelar lo absurdo de la evaluación dirigida en este sentido cuyos resultados positivos habrán de ser más prodo, cuyos resultados positivos habran de ser más proprogramado.

\section{BIBLIOGRAFÍA}

GARCÍA MANSO, J.M. (1999). La fuerza. Madrid: Gymnos.

HAHN, E. (1988). Entrenamiento con niños. Técnica, práctica y problemas específicos. Barcelona: Martínez Roca.

MEC (1991). R.D. 1006/1991, de 14 de junio, de en señanzas minimas correspondientes a la Educación Primaria. B.O.E. 152, 1991

MEC (2001). R.D. 3473/2000, de 29 de diciembre, de enseñanzas mínimas correspondientes a la Educación Secundaria.

NAVARRO VALDIVIELSO, F. (2001). Desarrollo, comportamiento motory deporte. Madrid: Síntesis. 